\section{Current Indications to Surgical Repair of the Aneurysms of Ascending Aorta}

Received: April 28, 2016; Accepted: April 29, 2016; Published: May 03, 2016

\section{Corresponding author: Nardi P}

\section{Background}

Normal ascending aorta functions as an elastic reservoir to enhance arterial flow. It stores energy during systole and dissipates it during diastole. The medial layer of the aorta consisting of elastin, collagen, and smooth muscle cells ensures the elasticity of the aortic wall. The ascending segment of the aorta is more elastic than the descending segment, due to its greater concentration of elastic fibres. Aortic aneurysm is defined as a permanent dilation of the aortic wall that exceeds 1.5 times the normal diameter of the aorta in a specified its segment (i.e., the segment of the ascending aorta has a normal value of 24-36 $\mathrm{mm}$, and its dilation for a diameter $>40 \mathrm{~mm}$ is called aneurysm).

Ascending aortic aneurysms are exposed to the risk of rupture with sudden death from cardiac tamponade, or dissection, i.e., acute, a dramatic event that can lead to cardiac tamponade, acute aortic valve insufficiency and severe ischemia of the heart, brain and abdominal viscera. The incidence of thoracic aortic aneurysms is approximately 6 cases per 100,000 people/year; male: female ratio is $2.4: 1$; the higher frequency is observed between the fifth and seventh decade of life [1].

Data of natural history of aortic aneurysms starts to the ' $80 \mathrm{~s}$. Bickerstaff et al. [2] reported in over 70 patients affected by not operated expansive or dissecting aneurysms a 5 year survival of $13 \%$ ( $19 \%$ for expansive aneurysms, $7 \%$ for dissecting) in comparison with 5 year survival of $75 \%$ observed in a normal population; $74 \%$ of patients had gone to rupture of the aneurysm, and of these $94 \%$ died.

Approximately, $50 \%$ of patients with acute untreated ascending aortic dissection die within $48 \mathrm{~h}$, and those undergoing emergency surgery have a $20-30 \%$ operative mortality. On the contrary, elective surgery lowers mortality to only 3-5\%. Understanding the pathophysiology of ascending aortic aneurysms can help to prevent and then to reduce mortality from aortic dissection or rupture, by indicating timely elective surgery.

\section{Indication to Surgical Repair of the Aneurysms of the Ascending Aorta}

Aneurysm diameter (size) is the main indication for elective surgical intervention, as it correlates strongly with the risk of the ascending aortic aneurysm to dissect or rupture, but indications for replacement of ascending aorta are importantly influenced ”pa.nardi4@libero.it

Cardiac Surgery Unit, Tor Vergata University Policlinic, Viale Oxford 81, Rome 00133, Italy.

Tel: $+39(06) 20903536$

Fax: +39 (06)20903538

Citation: Nardi P, Ruvolo G. Current Indications to Surgical Repair of the Aneurysms of Ascending Aorta. Journal of Vascular \& Endo Surgery. 2016, 1:2.

by etiology, rate of growth of the aneurysm, and family history of dissection.

\section{The diameter (size) of the aneurysm}

Coady et al. [3] in a study of several hundred patients with ascending aortic aneurysm found a relationship between size and risk of rupture of the aneurysm and/or dissection. In patients affected by an aneurysm of the ascending aorta with maximum transverse diameter $50-59 \mathrm{~mm}$ and in those with a diameter $>60 \mathrm{~mm}$ the risk of acute complications (rupture or dissection) during a 5 year follow-up was $17.8 \%$ and $27.9 \%$, respectively, in comparison with $9.5 \%$ for a diameter between 40 and $49 \mathrm{~mm}$. The risk of rupture increased therefore when the diameter reached $60 \mathrm{~mm}$. For these observations the authors recommend elective surgery when the diameter of the aneurysm reaches $55 \mathrm{~mm}$. In fact, the linear risk (per patient/year) of acute complications (rupture and/or dissection, death) for ascending aortic aneurysms $>60 \mathrm{~mm}$ was estimated at $15.6 \%$ vs. $6-8 \%$ for aneurysms with a diameter $(<60 \mathrm{~mm})[4,5]$.

\section{Etiological factors}

Patients with Marfan syndrome exhibit a more rapid growth of the aneurysm due to the characteristic collagenopathy, with higher risk of rupture or dissection already to a size of the aortic dilation smaller than that observed in presence of degenerative etiology, especially in the presence of family history of acute complications. Surgical treatment has improved the prognosis of these patients. In fact, over the years' 70 their life expectancy 
was 40 years: cause of death in $60-80 \%$ of cases was the rupture or acute dissection of the aorta, or heart failure from aortic regurgitation. By the mid-90s in the United States the average life of Marfan patients was 71 years, just under 73 years of age healthy population. This improvement was the result of the prevention (control of hypertension, excluding strenuous physical activity), the earlier diagnosis, and the elective early surgery.

Family syndromes of aortic aneurysm, not related to Marfan syndrome, i.e., in autosomal dominant gene transmission, $X$-linked or recessive, are at increased risk (about double) of annual growth and rupture.

In dissecting chronic aneurysms diameter growth is around six times greater than that of expansive aneurysms, probably due to the reduced resistance of the aortic wall dissected to the expansive intraluminal pressure.

An aortic valve disease which itself requires surgery does put more precociously the indication to the treatment of the aneurysm of the ascending aorta also already to a diameter $>45 \mathrm{~mm}$.

Bicuspid aortic valve represents the most common birth defect in the adult population, with a prevalence rate of $0.5-2 \%$. The aortic valve, which has only two cusps, not necessarily is malfunctioning; however, a normally functioning valve becomes in the years stenotic and/or insufficient. Aortic stenosis rarely leads to post-stenotic dilatation of the ascending aorta. Bicuspid valve indeed is associated with dilation of the root and/or the above-junctional ascending aorta, depending on the relation to a genetic defect linked to the activation of metalloproteinases, responsible for an abnormal structural weakening of the elastic fibers of the media, with cystic degeneration of the media similar to that observed in Marfan syndrome [6, 7].

The more recently published Guidelines recommend ascending aorta replacement a diameter $\geq 55 \mathrm{~mm}$ in absence of Marfan syndrome or other genetic-mediated disorders. On the contrary, patients affected by Marfan or Bicuspid valve-related aortopathy (as well as in presence of chronic dissection), should be referred to elective surgery earlier, when aneurysm dilation of the ascending aorta reaches $45-50 \mathrm{~mm}$, or $45 \mathrm{~mm}$ when patients have additional risk factors, i.e., size increase $>3 \mathrm{~mm} /$ year, family history of dissection, severe aortic regurgitation, or desire of pregnancy $[8,9]$.

However, thanks to improved surgical techniques and the use of prosthetic materials impervious to blood, surgical risk is very low and elective surgery in patients affected by degenerative expansive aneurysms can be performed when aortic diameter reaches $50 \mathrm{~mm}$, in order to eliminate the annual risk of rupture or acute dissection $[8,9]$.

Consequently, indications to replace ascending aorta in the clinical practice are as follows: 1) ascending aortic aneurysm $>5.0 \mathrm{~cm}$ in diameter; 2) ascending aortic aneurysm $\geq 4.5 \mathrm{~cm}$ in patients affected by Marfan syndrome or in presence of a bicuspid aortic valve; 3) ascending aortic aneurysm $>4.5 \mathrm{~cm}$ in patients undergoing aortic valve surgery; 4) growth rate of $>0.5$ $\mathrm{cm} /$ year when ascending aorta is $<5.0 \mathrm{~cm}$ in diameter. In any case, the surgical indication should be evaluated for each individual patient depending on risk factors, co-morbidities, body size, or significant increase of aortic size in short time. In addition, the study of genetic and molecular markers involved in the formation of the aneurysm can be helpful in decision making surgery. As recently published, in 108 patients subjected to ascending aorta replacement electively and in 26 treated for acute dissection, it was found that a particular phenotype (type III) of the aortic wall (i.e., elevated cystic medial degeneration without substitutive fibrosis) was similarly present either in a subgroup of expansive aneurysm than in all dissection aneurysms operated on, thus indicating that kind of phenotype as an optimal biomarker of dissection, independently of aneurysm diameter or aortic valve disorder [10]. In a study focused on the relation between ascending aorta dilation and presence of a bicuspid aortic valve (BAV), we found that in presence of BAV different genetic and molecular biomarkers, i.e., severe plurifocal apoptosis of smooth muscle cells and matrix metalloproteinases, were significantly involved in aneurysmal dilatation in comparison with the presence of a tricuspid valve. Identification of BAV at high risk of aneurysm development can be useful to differently treat this subset of patients, i.e., with a complete removal of the ascending aorta, including the aortic root with or without dilation [11].

\section{Surgical Procedures}

\section{Isolated ascending aorta replacement}

In the absence of dilation of the aortic root and aortic valve pathology, the aneurysmal segment of the ascending aorta above Sino-tubular junction is resected and replaced with a Dacron cylindrical tubular prosthesis, suitably shaped in order to avoid "kinking" of the tube into the concavity, by means of two continuous sutures of polypropylene for the proximal anastomosis and the distal end. The replacement of the ascending aorta with a tubular prosthesis also allows the reconstruction of the Sino tubular junction with re-suspension of the aortic valve cusps, in order to correct a failure from eccentric stretch of the commissures. To stabilize and improve this correction, it can be added aortic valve repair.

In Marfan patients the aortic root, although not dilated at the time of replacement of the supra-junctional ascending aorta, also needs to be replaced for the risk of subsequent expansion and need for further intervention.

\section{Separate aortic valve and ascending aorta replacement}

Ascending aortic aneurysms, with normal sinuses and aortic annulus, require replacement of the ascending aorta from the Sino-tubular ridge to the origin of the innominate artery with a Dacron tube graft similarly to the isolated ascending aorta replacement. In addition, the diseased aortic valve is replaced separately with a mechanical or biological prosthetic valve.

\section{Complete replacement of the ascending aorta}

Bentall-De Bono operation [12] is suggested to be a surgical "gold standard" for the treatment of aneurysms of ascending aorta involving the aortic root. This procedure can be done in presence of a significant dilation of the aortic root (diameter $>50$ $\mathrm{mm}$ for degenerative disease, or $\pm 45 \mathrm{~mm}$ in presence of bicuspid 
aortic valve, Marfan syndrome or other genetic disorders. In our experience, Bentall operation is performed also in presence of an asymmetrical dilatation of the aortic root (i.e., although the aortic root diameter does not reach $45 \mathrm{~mm}$, one or two of Valsalva coronary sinuses are affected by a marked dilation and/ or in presence of significant coronary ostium/ostia dislocation). All Marfan patients should undergo replacement of the ascending aorta including aortic root. The procedure is usually done with a composite graft consisting of a mechanical valve inserted into a collagen- or gelatin-impregnated preassembled Dacron tubular graft, or suturing on the tubular graft a biological prosthesis. Coronary artery ostia are reimplanted as buttons ("button technique").

\section{"Valve-Sparing" operations}

In the early $90 \mathrm{~s}$, after 30 years of replacement surgery, were proposed, in selected groups of patients, some techniques of conservative surgery ("valve-sparing") of the aortic valve, alternatively to the Bentall operation. These techniques have the advantage of avoiding complications related to the implant of a valvuar prosthesis, both mechanical (bleeding complications anticoagulation therapy-related) and biological (redo operation due to structural valve degeneration). The native aortic valve can be spared if, in presence of dilation of the Valsalva sinuses, the leaflets are normal and aortic insufficiency is secondary to dilation of the Sino-tubular ridge or aortic root, without structural valve alterations (i.e., calcifications, stenosis, marked prolapse of cusps, excessive diastases of cusps in presence of anulo-aortic ectasia). Two techniques have been proposed by Yacoub (aortic root "Remodeling") [13] and David (aortic valve "Reimplantation") $[14,15]$. Both interventions replace the ascending aorta with coronary ostia reimplantation on the Dacron tubular graft, while preserving the native valve, thus restoring its continence. Without implantation of a prosthetic valve, "valve-sparing" operations give the prospect of a better quality of life (not being required anticoagulation with its complications) and a lower risk of endocarditis. The major limit of these procedures is represented by the potential progression of aortic valve insufficiency during follow-up that may require a late redo operation.

\section{Our experience}

From January 2015 to February 2016, at the Cardiac Surgery Unit of the Tor Vergata University of Rome, on a total of five hundred and ninety-two open heart procedures, elective surgery on the ascending aorta was performed in 83 patients (15\%). Mean age of patients was $64 \pm 14$ years, mean EuroSCORE II $4.7 \%$.
Table 1 Surgical procedures on the ascending aorta.

\begin{tabular}{|cc|}
\hline Type of procedure & No. pts (\%) \\
$\begin{array}{c}\text { Ascending aorta replacement (supra-junctional) } \\
\text { +/- aortic valve sub-commissural valvuloplasty }\end{array}$ & $21(25)$ \\
\hline $\begin{array}{c}\text { Ascending aorta replacement+aortic valve } \\
\text { replacement }\end{array}$ & $12(15)$ \\
\hline $\begin{array}{c}\text { Bentall +/- associated procedures* } \\
50(60)\end{array}$ \\
\hline
\end{tabular}

*Mitral valve surgery, CABG

Table 2 Surgical times of different types of aortic repair procedure.

\begin{tabular}{lll}
$\begin{array}{l}\text { Type of aortic repair procedure } \\
\text { Ascending aorta replacement }\end{array}$ & $\begin{array}{l}\text { Cross-clamp } \\
\text { aortic time }\end{array}$ & $\begin{array}{l}\text { Cardiopulmonary } \\
\text { bypass time }\end{array}$ \\
$\begin{array}{l}\text { (supra-junctional) +/- aortic } \\
\text { valve sub-commissural } \\
\text { valvuloplasty, minutes (mean } \\
+/ \text { - SD) }\end{array}$ & $\begin{array}{l}58+/-26 \\
* * *\end{array}$ & $82+/-32^{* * *}$ \\
$\begin{array}{l}\text { Ascending aorta replacement } \\
+ \text { aortic valve replacement, } \\
\text { minutes (mean +/-SD) +/- } \\
\text { associated procedures }\end{array}$ & $100+/-27$ & $122+/-31$ \\
$\begin{array}{l}\text { Bentall +/- associated } \\
\text { procedures, minutes (mean } \\
+/- \text { SD) }\end{array}$ & $105+/-28$ & $125+/-32$ \\
\hline
\end{tabular}

$* p<0.0001$ vs. Bentall operation times

${ }^{* *} p<0.001$ vs. Ascending aorta repair associated with aortic valve replacement times

Bentall procedure was performed more frequently (50 cases, $60 \%$ ). There was no operative mortality; in 3 patients $(4.1 \%)$ postoperative course was complicated by low output syndrome, in 3 (4.1\%) by re-exploration for bleeding, in 1 (1.2\%) by need for pacemaker implantation, in $1(1.2 \%)$ by early endocarditis. Type of procedures and surgical times are summarized in Tables $\mathbf{1}$ and $\mathbf{2}$.

\section{Conclusion}

Ascending aortic aneurysm is a lethal disease [16, 17]. Elective surgical repair remains the gold standard for the management of symptomatic aneurysm or asymptomatic aneurysm with a diameter $\geq 5.5 \mathrm{~cm}[8,9]$. However, considering the low operative risk for elective surgery, in the current clinical practice and specific patient-related factors, such as molecular and genetic biomarkers involved in aortic aneurysm development [10,11], appropriate preventive surgery can be recommended in a timely manner (aortic diameter $>5.0 \mathrm{~cm}$ ). Lower threshold of aortic diameter for surgery should be considered for patients with aortopathy related to congenital disorders (i.e., Marfan syndrome, bicuspid aortic valve), and Bentall operation can be considered the gold standard surgical therapy in this subset of patient population. 


\section{References}

1 Brinster DR, Rizzo RJ, Bolman RM (2008) Ascending aortic aneurysms cardiac surgery in the adult. New York: McGraw-Hill pp: 1223-1246.

2 Bickerstaff LK, Pairolero PC, Hollier LH, Melton LJ, Van Peenen HJ, et al. (1982) Thoracic aortic aneurysms: A population-based study. Surgery 92: 1103-1108.

3 Coady MA, Rizzo JA, Hammond GL, Mandapati D, Darr U, et al. (1997) What is the appropriate size criterion for resection of thoracic aortic aneurysms. J Thorac Cardiovasc Surg 113: 476-491.

4 Coady MA, Rizzo JA, Elefteriades JA (1999) Developing surgical intervention criteria for thoracic aortic aneurysms. Cardiol Clin 17: 827-839.

5 Coady MA, Rizzo JA, Hammond GL, Kopf GS, Elefteriades JA (1999) Surgical intervention criteria for thoracic aortic aneurysms: A study of growth rates and complications. Ann Thorac Surg 67: 1922-1926.

6 Bonderman D, Gharehbaghi Schnell E, Wollenek G, Maurer G, Baumgartner $\mathrm{H}$, et al. (1999) Mechanisms underlying aortic dilatation in congenital aortic valve malformation. Circulation 99: 2138-2143.

7 Svensson LG, Crawford ES (1997) Degenerative aortic aneurysms Cardiovascular and Vascular Disease of the Aorta. Philadelphia WB Saunders.

8 Hiratzka LF, Bakris GL, Beckman JA, Bersin RM, Carr VF, et al. (2010) ACCF/AHA/AATS/ACR/ASA/SCA/SCAI/SIR/STS/SVM guidelines for the diagnosis and management of patients with thoracic aortic disease. Circulation 121: e266-369.
9 Erbel R, Aboyans V, Boileau C, Bossone E, Di Bartolomeo R, et al. (2014) ESC guidelines on the diagnosis and treatment of aortic diseases. Eur Heart J 35: 2873-2926.

10 Pisano C, Maresi E, Merlo D, Balistreri CR, Candore G, et al. (2012) A particular phenotype of ascending aorta aneurysms as precursor of type A aortic dissection. Interact Cardio Vasc Thorac Surg 15: 840846.

11 Balistreri CR, Pisano C, Candore G, Maresi E, Codispoti M, et al. (2013) Focus on the unique mechanisms involved in thoracic aortic aneurysm formation in bicuspid aortic valve versus tricuspid aortic valve patients: Clinical implications of a pilot study. Eur J Cardiothorac Surg 43: e180-e186.

12 Bentall HH, De Bono A (1968) A technique for complete replacement of the ascending aorta. Thorax 23: 338-339.

13 Sarsam MA, Yacoub MH (1993) Remodeling of the aortic valve annulus. J Thorac Cardiovasc Surg 105: 435-438.

14 David TE, Feindel CM (1992) An aortic valve-sparing operation for patients with aortic incompetence and aneurysm of the ascending aorta. J Thorac Cardiovasc Surg 103: 617-621.

15 David TE (2003) Aortic valve repair and aortic valve-sparing operations Cardiac Surgery in the Adult. New York: McGraw-Hill pp: 811-824.

16 Saliba E, Sia Y (2015) The ascending aortic aneurysm: When to intervene. IJC Heart \& Vasculature 6: 91-100.

17 Nardi P, Russo M, Saitto G, Pellegrino A, Ruvolo G (2015) Surgical treatment of the ascending aorta aneurysm associated to bicuspid aortic valve. Edorium J Surg 2: 6-8. 